\title{
L'attribution de la qualité de preuve - une action négligée
}

La fonction de la preuve est centrale dans le processus de renversement de la présomption d'innocence. Or, dans un premier temps, il n'y a pas de preuve mais que des traces. Partant, cette contribution critique que l'on accorde d'un point de vue dogmatique, législatif et jurisprudentiel trop peu d'attention à cette action normative de transformation ou de qualification des éléments recueillis en preuve. Depuis la création du CPP, la distinction entre l'administration et l'exploitation des preuves passe du flou à l'obscurité totale - ce qui est démontré dans cette contribution d'un point de vue dogmatique d'abord puis en pratique par rapport à trois domaines spécifiques : les éléments recueillis par la police, par le Service de renseignement et par les personnes privées à l'interne des entreprises.

\section{Introduction}

II. Qualifier un élément recueilli en tant que preuve

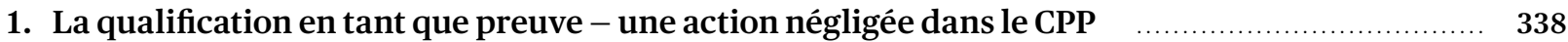

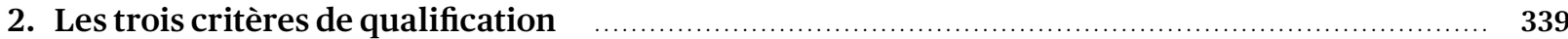

3. Trois domaines provoquant un passage du clair au flou à l'obscurité totale $\ldots \ldots \ldots \ldots \ldots \ldots \ldots \ldots . \ldots \ldots$

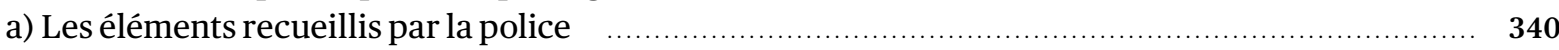

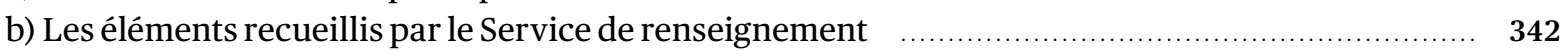

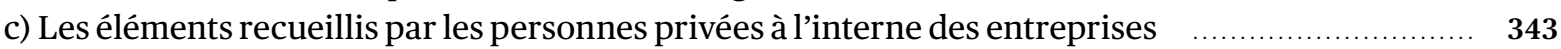

Citation:

NADJA CAPUS, L'attribution de la qualité de preuve une action négligée, sui generis 2020, S. 337

Prof. Nadja Capus, Professeure ordinaire de droit pénal et procédure pénale à l'Université de Neuchâtel (nadja.capus@unine.ch). Il s'agit d'une partie de la contribution Le régimejuridique des preuves du projet initial à la réalité d'aujourd'hui : du flou à l'obscuritétotale à l'ouvrage « Dix ans de Code de procédure pénale » (Bohnet/Dupont/Kuhn, édit.), Neuchâtel 2020 (à paraître et à commander sur le site Internet https://publications-droit.ch). L'auteure remercie son assistante A. Beretta pour la relecture, un peer reviewer de sui generis anonyme, le professeur Marc Thommen ainsi que le Dr G. Mégevand, procureur fédéral, pour leurs commentaires.

URL: sui-generis.ch/142

DoI: https://doi.org/10.21257/sg.142

Ce(tte) œuvre est mise à disposition selon les termes de la Licence Creative Commons Attribution - Partage dans les Mêmes Conditions 4.0 International. 


\section{Introduction}

1 Le législateur fédéral a une aversion sympathique contre des lois trop détaillées. Lors de l'élaboration du Code de procédure pénale fédéral (CPP) par exemple, le professeur Niklaus Schmid, auteur de l'avant-projet, s'est même vu interrogé par le Département fédéral de la Justice sur la quantité d'articles. La question qui se posait était de savoir si on avait « vraiment besoin de réglementer autant dans le détail $» 1$.

2 Le Code finalement adopté et actuellement en vigueur contient environ 60 articles (art. $10 \mathrm{al} .2 \mathrm{CPP}$ et les articles 139-196 CPP, art. 343 CPP, etc.) concernant les moyens de preuve. De plus, le législateur helvétique s'est positionné à l'époque en tant que pionnier en introduisant l'art. 141 CPP, qui aborde l'inexploitabilité de certaines preuves ${ }^{2}$.

3 Toutefois, cette contribution part de l'hypothèse qu'il y a une partie négligée : celle de l'administration des preuves. Pour expliquer cette démarche, j’ose partager une expérience personnelle. En effet, au départ, lorsque j'ai commencé à travailler en français en 2016, j'ai eu du malà bien saisir le sens donné à cette notion en français. Il me semble important de l'évoquer pour souligner la transposition de la même action en allemand : Beweiserhebung. La notion allemande fait ainsi ressortir l'existence d'une action intermédiaire entre le recueil de tous les éléments (informations, documents, objets) susceptibles d'être pertinents pour une enquête et leur exploitation en tant que preuve. Autrement dit, parmi tous les éléments recueillis par l'autorité de poursuite, seuls quelques-uns seront érigés ou transformés en preuve. Par conséquent, il se peut que des éléments recueillis ne soient pas transformés, voire même transformables en preuve. Le fait d'écarter un élément recueilli à ce stade déjà a pour effet que la question ultérieure d'(in)exploitabilité d'une preuve ne se pose même pas. Nous allons ainsi aborder, dans un premier temps, les bases légales et le traitement jurisprudentiel consacrés à cette transformation que nous appelons la qualification des éléments en tant que preuve (II.).

4 Pour éviter toute confusion sémantique, on utilisera par ailleurs la notion « moyen de preuve» pour désigner uniquement le procédé utilisé pour obtenir une preuve ou, pour reprendre l'art. $140 \mathrm{CPP}$, la méthode d'administration de la preuve (audition, expertise, etc.), tandis que la " preuve » se matérialise dans le résultat de ce procédé

1 Office fédérale de la justice, De 29à l'unité, Informations sur le futur Code de procédure pénale suisse et la procédure pénale des mineurs, Berne mars 2001, p. 2.

2 SABINE GLESS, Sinn und Unsinn von Beweisverwertungsverboten strafprozessuale Wahrheitssuche und ihre Grenzen im Rechtsvergleich, RPS 137/2019, p. 5. (procès-verbal d'audition/déclarations, rapport d'expertise, etc.). En ce sens, le titre de l'art. $141 \mathrm{CPP}$ est trompeur, puisqu'il devrait mentionner l'exploitation des preuves et non des moyens de preuves. Cette confusion se retrouve aussi dans la doctrine et la jurisprudence.

Pour terminer, nous allons tirer notre conclusion quant 5 à la manière dont le législateur, la doctrine et la jurisprudence procèdent à la qualification en tant que preuve et à la disqualification (III.).

\section{Qualifier un élément recueilli en tant que preuve}

\section{La qualification en tant que preuve - une action négligée dans le CPP}

Comme évoqué précédemment, il existe une action peu 6 abordée en doctrine et en jurisprudence : la qualification en tant que preuve. C'est plutôt l'exploitation de la preuve qui a su attirer toute l'attention alors que cette question ne se pose qu'une fois que l'élément recueilli a été qualifié en tant que preuve.

Faire la distinction entre ces deux étapes s'impose d'un 7 point de vue légal (cf. à ce propos également le sous-chapitre II.2. ci-après) ainsi que d'un point de vue logique. En fait, il n'y a pas de preuve au sens strict qui puisse être recueillie lors d'une enquête, mais seulement des informations, des objets, des traces forensiques, etc., car la notion de " preuve " présuppose une attribution normative d'une fonction précise - attribution qui ne peut être effectuée que si certaines conditions sont remplies ${ }^{3}$.

La question de l'attribution de la qualité de preuve n'est 8 toutefois pas inconnue en droiz suisse, mais la discussion qui y est consacrée n'est menée que de manière restreinte. En effet, elle ne cible que l'expertise privée réalisée sur mandat du prévenu : le Tribunal fédéral nie sa transformabilité en preuve au sens propre et refuse ainsi de lui attribuer la qualité d'une preuve ${ }^{4}$.

3 LAI HO HOCK, The Legal Concept of Evidence, Stanford Encyclopedia of Philosophy, Stanford 2015 ; LEE LOEVINGER, Facts, Evidence and Legal Proof, Western Reserve Law Review 2/1958, p. 155 s.

4 ATF 141 IV 369 consid. 6.2 ; Tribunal cantonal du Fribourg 5022018 134 du 7. mai 2019 consid.3.2.3 ; MARIANNE HEER, in: Niggli/Heer/ Wiprächtiger (édit.), Basler Kommentar, Schweizerische Strafprozessordnung/Schweizerische Jugendstrafprozessordung (StPO/ JStPO), $2^{\mathrm{e}}$ éd., Bâle 2014, art.182 N 10 et art.189 N 6 (cit.: BSK StPOAUTEUR); WOLFGANG WOHLERS, in: Donatsch/Hansjakob/Lieber (édit.), Kommentar zur Schweizerischen Strafprozessordnung (StPO), 2 e éd., Zurich 2014, art.182 N15 (cit.: Donatsch/Hansjakob/ Lieber-Kommentar-AUTEUR); NIKLAUS SCHMID / DANIEL JOSITSCH, Schweizerische Strafprozessordnung - Praxiskommentar, $3^{\mathrm{e}}$ éd., Zurich/St-Gall 2017, art.182 N 7. Cf. pour la critique de cette jurisprudence ci-après N 45 ss. 
9 Le fait qu'il s'agisse d'une action distincte de l'exploitation ressort du CPP lui-même. Le sous-chapitre du $4^{\mathrm{e}}$ titre dédié aux moyens de preuve contient, comme première section du premier chapitre, les deux notions : administration et exploitation. Deux des trois dispositions de cette section traitent de l'administration : l'art. 139 al. 1 (en utilisant « mettre en œuvre » / « einsetzen » / « avvalersi » comme synonymes selon nous) et al. 2 ainsi que l'art.140 CPP. Ils limitent l'administration en exigeant certains critères (art. 139 al. 1 CPP), en excluant la nécessité (art.139 al. 2 CPP) ou certaines méthodes de prélèvement (art.140 CPP). lés dans le Code qui mentionnent cette action d'administration : les art. 3 let. d, 131 al. 3, 141 al. 4, 147, 223, 311 al. 1, 313, $318 \mathrm{al}$. 2, $332 \mathrm{al}$. 3, $388 \mathrm{~s}$. CPP. Ils règlent le processus de l'administration : comment il (ne) faut (pas) procéder, qui doit ou peut y participer et quand peut-on procéder à l'administration.

11 Nous allons maintenant focaliser notre analyse sur les critères qui découlent du CPP et dont la qualification d'un simple élément recueilli en tant que preuve dépend.

\section{Les trois critères de qualification}

12 Selon l'art. 139 al. 1 CPP, qui est la disposition principale concernant les preuves,

« [l]es autorités pénales mettent en œuvre tous les moyens de preuves licites qui, selon l'état des connaissances scientifiques et l'expérience, sont propres à établir la vérité. ".

13 Il résulte de cette disposition que le législateur rattache deux critères à la qualification en tant que preuve d'un élément recueilli par l'autorité de poursuite. Il y a le critère de la licéité, qui se confond, selon le cadre normatif imposé par le législateur, avec les critères d'exploitabilité (cf. partie III), ainsi que celui de l'aptitude de l'élément à prouver la vérité matérielle. Cette aptitude est constituée par la validité, la fiabilité ainsi que la force de persuasion de l'élément recueilli ${ }^{5}$.

\footnotetext{
5 HENRY LÉVY-BRUHL, La preuve judiciaire : Étude de sociologie juridique, Paris 1964, p. 22 : « Prouver, c'est faire approuver » ; cf. également BSK StPO-GLESS, art. 139 N15 ; à propos de l'accessibilité, la fiabilité et le problème d'interprétation des moyens de preuve techniques, cf. JOËLLE VUILLE / JÜRG ARNOLD, L'appréciation des preuves techniques en matière de circulation routière - les traces numériques, Circulation routière $3 / 2019$, p. 60 ss.: la partie non interprétable du code devrait, à leur sens, être écartée du dossier car elle ne remplit pas les conditions de l'art.139al.1CPP ; cf. le projet de recherche dirigé par la Professeure Joëlle Vuille (FNS n ${ }^{176720)}$ qui vise à investiguer comment la loi au sens large encadre le recours aux preuves de nature scientifique afin d'en assurer la fiabilité.
}

Or, il y a un troisième critère auquel il devrait être tenu 14 compte dans le contexte de l'art.139 CPP : le critère supplémentaire de la documentation. En fait, le principe de base impose à l'autorité de documenter tout ce qui n'est pas originairement accompli en la forme écrite dans un dossier, lequel doit obligatoirement être constitué pour chaque affaire pénale ${ }^{6}$.

Cette obligation fondamentale est le corollaire néces- 15 saire du droit d'accéder au dossier ${ }^{7}$ - un droit qui constitue une condition préalable incontournable pour garantir le droit à la défense ${ }^{8}$. Dès lors, tant le droit à un procès équitable ${ }^{9}$ que le droit d'être entendu ${ }^{10}$ y sont liés.

Le Tribunal fédéral a précisé que cette obligation de do- 16 cumentation ne touche pas seulement l'existence d'un élément en soi, mais également sa production, soit la manière dont il a été recueilli11. Autrement dit, la production des éléments, potentiellement transformables en preuve ultérieurement, doit être intelligible, traçable ${ }^{12}$. Ceci vaut pour tous les éléments recueillis qui pourraient se révéler importants dans la procédure et ainsi transformés en preuves, même si ce n'est qu'avec une faible probabilité. En effet, les autorités chargées de l'enquête et du procès ont l'obligation d'intégrer dans le dossier tous les éléments recueillis ayant un lien avec l'affaire ${ }^{13}$.

Nous pouvons donc constater que la question de savoir 17 si de tels éléments sont marqués d'un défaut matériel (en termes de contenu) ou de forme ainsi que, le cas échéant, la question de savoir si ces défauts donnent lieu à des objections quant à leur exploitabilité ne devrait être posée que dans un deuxième temps ${ }^{14}$. Cela signifie qu'il faut d'abord vérifier si les dossiers sont complets et s'ils contiennent toutes les informations nécessaires qui permettent de reconstruire l'obtention des éléments susceptibles d'être transformés en preuve. En cas d'omis-

6 Cf. art. 100 al. 1 et 76 al. 1 CPP ; YVAN JEANNERET / ANDRÉ KUHN, Précis de procédure pénale, $2^{\mathrm{e}}$ éd., Berne 2018, N 5010.

7 Cf. art. 3 al. 2 let. c et 107 al. 1 let. a CPP ; BSK StPO-SCHMUTZ, art. 100 N1 ; JEANNERET/KUHN, N4O27 ss sur les restrictions possibles.

8 Art. 32 al. 2 Cst.

9 Art. 29 al. 1 Cst., art. 6 chiff. $1 \mathrm{CEDH}$.

10 Art. 29 al. 2 Cst., art. 6 chiff. 3 CEDH.

11 Décision du tribunal fédéral 6B_1368/2017 du 14 juin 2018 consid. 2.3 « In einem Strafverfahren bedeutet dies, dass die Beweismittel, jedenfalls soweit sie nicht unmittelbar an der gerichtlichen Hauptverhandlung erhoben werden, in den Untersuchungsakten vorhanden sein müssen und dass aktenmässig belegt sein muss, wie sie produziert wurden, damit die beschuldigte Person in der Lage ist zu prüfen, ob sie inhaltliche oder formelle Mängel aufweisen und gegebenenfalls Einwände gegen deren Verwertbarkeit erheben kann. »

12 Décision du tribunal fédéral 6B_1368/2017 du 14 juin 2018 consid. 2.3 ; BSK StPO-GLESS, art.139 N16.

13 Décision du tribunal fédéral 6B_1368/2017 du 14 juin 2018 consid. 2.3.

14 Cf. déjà ATF 129 I 85 consid. 4.1. - à l'époque encore sur la base du droit cantonal. 
sion de la police ou du ministère public de faire ressortir du dossier la source d'un élément recueilli, c'est sur demande d'une partie ou du juge que l'omission pourra être réparée sans que cela n'affecte $a b$ initio l'aptitude de l'élément à être utilisé comme preuve.

En revanche, il est exclu de parler de preuve concernant des éléments sans une telle trace documentée : ils ne peuvent de toute manière pas être transformés en une preuve et ainsi le juge ne peut pas s'appuyer sur un tel élément pour établir la vérité matérielle, comme ce serait le cas pour une preuve. On peut se demander si on devrait alors lui accorder le statut d'indice : cas échéant l'élément ne serait dès lors pas écarté du dossier et la juxtaposition des ces informations pourra permettre au juge, par une déduction logique, de considérer un fait comme établi.

19 De toute manière, dans une telle constellation, la question de l'(in)exploitabilité en tant que preuve ne se pose donc même pas.

\section{Trois domaines provoquant un passage du clair au flou à l'obscurité totale}

20 Nous allons maintenant analyser l'attribution de la qualité de preuve aux éléments recueillis dans trois contextes délicats.

\section{a) Leséléments recueillis par la police}

21 Il est incontesté que l'obligation générale de documentation selon l'art. 76 al. 1 en lien avec l'art. 100 al. 1 CPP s'applique à tous les stades de la procédure. En conséquence, elle s'applique également dans la procédure d'investigation policière. Cette phase - faisant partie de la procédure préliminaire - est également soumise aux garanties fondamentales de procédure, indépendamment de la question de savoir si la police agit sur mandat ou si elle enquête de sa propre initiative ${ }^{15}$.

Étonnamment, le Tribunal fédéral est même allé jusqu'à qualifier le rapport de police en tant que tel comme preuve. Apparemment, notre Haute Cour a faussement interprété le lien étroit entre la qualification comme preuve et l'obligation de documenter, avec pour résultat la conclusion inverse selon laquelle toutes les pièces contenues dans un dossier (art. 100 al. 1 let. b CPP) sont $d e$ facto des preuves ${ }^{\mathbf{1 6}}$. Ainsi, le Tribunal fédéral a qualifié le rapport de police comme étant une preuve en soi en raison du fait que la police est une autorité pénale (art. 12 let. a et 15 CPP) qui est obligée de réunir des pièces dans un

15 MARC HENZELIN / SONJA MAEDER MORVANT, in: Jeanneret/Kuhn/ Perrier Depeursinge (édit.), Commentaire Romand, Code de procédure pénale suisse, CPP, $2^{\mathrm{e}}$ éd., Bâle 2019, art.15 N16 (cit. : CR CPP-AUTEUR).

16 Décision du tribunal fédéral6B_1057/2013 du19 mai 2014 consid. 2.3. dossier ${ }^{17}$. Cette conclusion est, bien évidemment, incorrecte car bien que le rapport de police mentionne, par exemple, des procès-verbaux d'interrogatoires ou des traces trouvées et conservées, cela ne saurait avoir pour effet de le transformer automatiquement en preuve ${ }^{18}$.

Malheureusement, malgré les règles explicites selon les- 23 quelles, premièrement, l'autorité pénale est soumise à l'obligation de documentation et, deuxièmement, les éléments recueillis ne peuvent être transformés en preuve que s'ils sont suffisamment documentés, le législateur a préféré pour le reste de la réglementation, passer au flou, puis à l'obscurité totale.

Il existe, par exemple, une démarcation en soi déjà vague 24 entre l'investigation policière dans le cadre de l'activité de police judiciaire et la collecte des informations en tant que police de sûreté ${ }^{19}$. Cette obscurité concernant la démarcation touche à l'application de l'obligation de documentation. Il est, par exemple, constaté dans la littérature que la police tenterait de se soustraire à cette obligation, notamment lorsqu'il s'agirait de documenter l'existence et la source d'un premier soupçon. Elle rédigerait ainsi des rapports dissimulés (verdeckte Rapportierung) dans une première phase d'enquête ${ }^{20}$.

Les rapports de police contiennent par exemple de va- 25 gues références telles que : « les enquêtes de police ont révélé.... ", " une référence a révélé... ».

Face à cette pratique, la doctrine a commencé à se poser 26 la question de savoir si et à quelles conditions de telles informations - recueillies durant l'enquête policière et dans le cadre de la fonction de police de sûreté - peuvent être transformées ensuite en preuve ${ }^{21}$.

Constatant l'absence de distinction entre administration 27 et exploitation de la preuve, il est proposé dans la littérature d'ajouter un alinéa à la disposition qui règle l'exploitation (un quatrième à l'art. $141 \mathrm{CPP}$ ) pour pallier au moins le fait qu'une base légale dans le CPP fait défaut lorsque des éléments sont recueillis par la police

\section{Ibid.}

18 Au moins, le tribunal fédéral requiert en cas d'un rapport de police contesté que celui-ci ne peut être pris en compte que si l'agent chargé du rapport a été confronté à l'accusé et ses questions à ce sujet (ibid., consid. 2.4).

19 ATF 136 I 87 consid. 3.4 ; ATF 143 I 27 consid. 2.5 ; DONATSCH/HANSJAKOB/LIEBER-Kommentar-KELLER, art.15N13 ; BSK StPO-RHYNER, art. $306 \mathrm{~N} 5 \mathrm{sS}$.

20 DONATSCH/HANSJAKOB/LIEBER-Kommentar-KELLER, art. 15 N14; LUKAS BÜRGE, Polizeiliche Ermittlung und Untersuchung, Charakteristik, Abgrenzungen und Auswirkungen auf die Beschuldigtenrechte, Berne 2018, p. 49.

21 BSK StPO-GLESS, art.141 N 38 ; SVEN ZIMMERLIN / MARCO GALELLA, Aspekte der beweismässigen Verwertbarkeit von polizeirechtlich erhobenen Informationen im Strafverfahren, FP 2019, p. 374 ss. 
hors du cadre d'une procédure pénale mais conformément à la loi (de la police) ${ }^{22}$. Lorsqu'ils sont recueillis en violation de la loi, c'est aux cantons de régler le sort de ces éléments ${ }^{23}$.

Selon notre approche, cependant, il ne peut de toute manière pas s'agir de preuve (et la question d'exploitabilité ne se poserait ainsi même pas) si l'existence et la production de l'information recueillie par la police ne sont pas suffisamment documentées.

Ceci est également valable si le critère principalement appliqué par la doctrine et la jurisprudence pour procéder à une démarcation entre l'enquête policière selon une loi de police cantonale et l'investigation policière selon le CPP, ferait défaut : le premier soupçon ${ }^{24}$. Une notion en soi flou, malgré son importance ${ }^{25}$.

30 En effet, afin que cette activité policière soit soumise le plus tôt possible au contrôle du ministère public et au respect des garanties judiciaires du CPP, la doctrine opte pour un seuil très bas et est encline à affirmer assez tôt l'existence d'un soupçon (suffisant selon l'art. 197 al. 1 let. b ou grave selon l'art. $269 \mathrm{al}$. 1 let. $\mathrm{a}^{26}$ ou encore fort selon l'art. 207 al. 1 let. d et l'art. 221 al. 1 CPP) ${ }^{27}$.

31 Il est parfaitement justifié que la doctrine et la jurisprudence ne requièrent pas un niveau de précision et de détail très élevé relatif au premier soupçon, notamment

22 ZIMMERLIN/GALELLA (n. 21), p.380 : «Informationen, die aus gesetzeskonform erfolgten polizeirechtlichen Massnahmen resultieren, dürfen als Beweise im Strafverfahren verwertet werden. ».

23 Il y a apparemment des lois cantonales, qui, par voie de simplification, renvoient à l'article $141 \mathrm{CPP}$, applicable à titre de droit cantonal supplétif. Tel est le cas par exemple des cantons de Vaud et du Valais, cf. CR CPP-BÉNÉDICT, art.141 N 7d.

24 Ni le CPP ni une autre loi fédérale ne contient aucune disposition sur laquelle peut se fonder l'action policière lorsqu'il n'y a pas encore de soupçons concrets d'infraction (PC LAURENT MOREILLON / AUDE PAREIN-REYMOND [édit.], Petit commentaire CPP, 2 é éd., Bâle 2016, art. 298b N 5); cf. JOSIANNE MAGNIN, Die Polizei : Aufgaben, rechtsstaatliche Grenzen und Haftung, Zurich et al. 2017, p. 29 s. ; THOMAS HANSJAKOB, Grenzen und Rahmenbedingungen der verdeckten präventiven Tätigkeit der Polizei, FP 2015 I, p. 34 ; GIANFRANCO ALBERTINI / BRUNO FEHR / BEAT VOSER, Polizeiliche Ermittlung: Ein Handbuch der Vereinigung der Schweizerischen Kriminalpolizeichefs zum polizeilichen Ermittlungsverfahren gemäss der Schweizerischen Strafprozessordnung, Zurich et al. 2008, p. 545 ; TANJA KNODEL, Verdacht und Verdachtsgrade, Revue de l'avocat 2019, p. 320 ss. ; HANS GIGER, Beweisverwertungsverbot im Strassenverkehrsrecht, Circulation routière 2/2019, p. 4 ss. ; ATF 140 I 353 consid. 5.1 ; ATF 143 IV 27 consid. 2.5 ; Décision du tribunal fédéral 6B $1143 / 2015$ du 6 juin 2016 consid. 1.3.1.

25 BÜRGE (n. 20), p. 41 ss.

26 Cf. Décision du tribunal fédéral 1B_230/2013 du 26 juillet 2013 consid. 5.1.

27 ATF 140 I 353 consid. 5.1 ; GREGOR WEY, Der Anfangsverdacht als Abgrenzungskriterium zwischen den kantonalen Polizeigesetzen und der StPO, Quid ? Fribourg Law Review 2019, p. 20 ; DONATSCH/ HANSJAKOB/LIEBER-Kommentar-HANSJAKOB, art. 298b N 11. lorsque la procédure se trouve encore dans une phase précoce. Le degré de certitude peut ainsi être relativisé en fonction de l'étape de la procédure pénale ${ }^{28}$.

Ce qui ne devrait cependant pas être relativisé sont les 32 exigences concernant la documentation ${ }^{29}$. En effet, l'autorité qui doit ordonner ou autoriser une mesure de contrainte liée à la récolte des éléments ou qui doit ultérieurement procéder à un contrôle judiciaire des mesures de contrainte exécutées, n'est pas en mesure de vérifier l'existence d'un tel soupçon - soupçon qui est une condition essentielle pour toute mesure de contrainte $^{30}$ - qu'à la condition que les éléments recueillis sont inclus dans le dossier. Ce n'est dès lors que des éléments transformés en tant que preuves qui vont permettre de prouver l'existence du premier soupçon et la manière dont il a été généré ${ }^{31}$.

Ainsi, selon notre approche, le point sur lequel l'autorité 33 doit se focaliser n'est ni le degré de certitude que la preuve doit atteindre par rapport à l'existence d'un éventuel soupçon, ni la question de savoir s'il s'agit d'une preuve illicite, ou d'une preuve illicite qui pourrait être toutefois ou néanmoins exploitable. La question à laquelle il faut répondre est tout d'abord de déterminer s'il s'agit d'une preuve tout court. Il n'y a pas, en effet, de preuve selon l'art. 139 al. 1 en lien avec les art. 100 al. 1 et $76 \mathrm{al} .1$ et $192 \mathrm{CPP}$ si la documentation de son existence et sa production font défaut ${ }^{32}$.

28 CR CPP-VIREDAZ/JOHNER, art. 197 N 5 ; les autres deux critères influençant le seuil du degré du soupçon sont la durée et la sévérité de la mesure de contrainte, cf. BSK StPO-wEBER, art. 197 N 8 et 8 a.

29 BÜRGE (n. 20), p. 49 s.

30 Cf. art.197 al. 1 let. b CPP.

31 DONATSCH/HANSJAKOB/LIEBER-Kommentar-KELLER, art.197 N 7 ; or, dans ce contexte encore, l'utilisation dans la litérature et jurisprudence des notions est confuse : le législateur, par exemple, utilise dans l'art. 225 al. 4 CPP - en permettant au tribunal des mesures de contrainte de recueillir les preuves immédiatement disponibles susceptibles de confirmer ou d'écarter les soupçons et les motifs de détention - la notion " preuve ", tout comme le Tribunal pénal fédéral lorsqu'il fait constamment référence à la situation de preuve (Beweislage) concernant la distinction entre soupçon simple et fort, cf. Décision tribunal pénal fédéral BV.2010.78 du 28. janvier 2011 consid. 2.2. D'autres sources parlent des indices (CR CPP-VIREDAZ/JOHNER, art. 197 N 5 ; ATF 141 IV 87 consid. 1.3.1 ; CORNELIA HÜRLIMANN, Die Eröffnung einer Strafuntersuchung im ordentlichen Verfahren gegen Erwachsene im Kanton Zürich, Zürich 2006, p. 107 s. ; NIKLAUS SCHMID / DANIEL JOSITSCH, Handbuch des schweizerischen Strafprozessrechts, $3^{\mathrm{e}}$ éd., Zurich/St-Gall 2017, p. 550 ; Décision du tribunal fédéral 6B_830/2013 du 10 décembre 2013 consid. 1.4).

32 GUGLIELMO PALUMBO / ANDRÉ KUHN, Projet Innocence Suisse - ou lorsque la justice pénale condamne à tort, Revue de l'avocat 2020 p. 85 ; cf. décision du Obergericht Zurich SB160065 du 17 juin 2016 consid. 3.4.6.1 et 3.4.6.3 concernant l'exigence de la documentation-sans toutefois en déduire que la qualité de moyen de preuve fait défaut, mais en procédant par le raisonnement décrit auparavant : moyen de preuve illicite car violation de l'art. 141 al. 2 CPP avec conclusion de l’inexploitabilité après avoir pesé les intérêts, cf. consid. 3.4.10 et 3.4.10.1. 

quelle rigiditél'obligation de documentation devrait être exigée. Une application assez vague semble être acceptée lorsque le stade de la procédure est encore précoce : il est admis que le Tribunal des mesures de contrainte peut tenir compte des éléments constatés par la police dans ses rapports, même si ceux-ci ne peuvent pas être davantage étayés ${ }^{33}$. Il est clair qu'une telle tolérance envers des éléments avec un défaut de transparence par rapport à leur production, sape le pouvoir de contrôle du Tribunal de mesure de contrainte - qui, en soi, n'est déjà pas de grande portée dans la pratique ${ }^{34}$. de documentation y lié sont exacerbés depuis quelques années suite à deux évolutions : premièrement, suite au renforcement de l'activité préventive-répressive de la police, par exemple par lesmodifications apportées ces dernières années à plusieurs lois cantonales de police, modifications qui étendent les possibilités policières de rechercher et recueillir des informations ${ }^{35}$. Cette activité policière de caractère préventive-répressive est aussi due à l'évolution technique qui permet, entre autres, l'utilisation des logiciels de recherche qui se basent sur des algorithmes (Predpol, Precobs) et qui génèrent une quantité d'informations jamais vues auparavant ${ }^{36}$. Deuxièmement, le législateur a commençé à inclure dans le CPP des mesures de poursuite pénale à caractère préventif et proactif : les art. $255 \mathrm{ss} \mathrm{CPP}$, les art. 260 ss CPP, l'art. 237 al. 2 let. a et g СРP37.

\section{b) Leséléments recueillis par le Service de renseignement}

36 Dans ce sous-chapitre, nous allons aborder si et à quelles conditions l'intégration des informations recueillies par le Service de renseignement de la Confédération (ciaprès : le SRC) est acceptée dans notre ordre juridique en tant que preuve dans une procédure pénale.

37 A titre de rappel : dans le cadre d'une enquête menée par le SRC, il est accordé beaucoup de libertés et d'intrans-

33 Comme par ex. l'identité d'un informateur, cf. ATF 142 IV 289 consid. 2.2.3.

34 DIEGO R. GFELLER / OLIVIA SIEBER, Zwangsmassnahmengerichte : Thanks for nothing ?, Revue de l'Avocat 2019, pp.329 ss.

35 Cf. la correction (restriction des pouvoirs intégrés) du tribunal fédéral de deux lois de police cantonale : ATF 140 I 353 (Zurich) et décision du tribunal fédéral 1C_181/2019 du 29 avril 2020 (Berne).

36 NADJA CAPUS, Des algorithmes à risque dans la justice pénale, Plaidoyer 6/2018, p. 20 ss ; ATF 145 IV 42 consid. 4.2.

37 Cf. ZIMMERLIN/GAIELLA (n. 21), p. 378 pour la Suisse ; pour l'exemple des Pays-Bas, cf. JILL E.B. COSTER VAN VOORHOUT, Intelligence as legal evidence: Comparative criminal research into the viability of the proposed Dutch scheme of shielded intelligence witnesses in England and Wales, and legislative compliance with Article 6 (3) (d) ECHR, Utrecht Law Review 2/2006, p.119 ss., 121 ss. parence compte tenu du but qui tient dans la sauvegarde d'intérêts nationaux importants tels qu'ils sont mentionnés aux art. 2 et 3 de la Loi fédérale sur le renseignement (LRens).

Le prix à payer serait, en principe, le respect d'une mu- 38 raille de Chine entre les enquêtes menées sur la base de la LRens et les procédures pénales selon le CPP - mur qui s'est cependant écroulé depuis les événements du $11 \mathrm{sep}$ tembre 2001 aux Etats-Unis. Le fait de fixer des limites très strictes au traitement d'informations avant une poursuite pénale engendrait un certain risque au niveau de la sécurité, ce qui ne semblait plus acceptable ${ }^{38}$.

On nous a assuré à plusieurs reprises dans le Message 39 concernant l'introduction de la loi en vigueur aujourd'hui, que le SRC ne devrait pas assumer un rôle de police auxiliaire ${ }^{39}$; que les activités préventives du SRC doivent être clairement distinguées des activités répressives des autorités de poursuite pénale ; que le SRC n'assure pas de tâches de police ou de procédure pénale ; que les activités du SRC et des autorités de poursuite pénale sont complémentaires, mais que les unes ne sont pas le préliminaire des autres, raison pour laquelle leur surveillance relève de domaines différents (le SRC par les instances politiques, les autorités de poursuite pénale par les tribunaux). En conséquence, l'échange d'informations entre le SRC et les autorités de poursuite doit de ce fait obéir à des règles précises ${ }^{40}$.

Bien que, évidemment, seule une séparation précise et 40 stricte soi conforme à un Etat de droit, non seulement l'échange des informations entre les différentes autorités, notamment entre le Ministère public de la Confédération et le SRC est renforcé depuis quelques années ${ }^{41}$, mais également l'intégration des informations recueillies par le SRC en tant que preuve dans une procédure pénale est largement acceptée dans notre ordre juridique $^{42}$. Le SRC identifie des personnes qui représenteraient une menace concrète pour la sécurité intérieure et extérieure de la Suisse. Il envoie ses rapports avec de telles informations aux autorités pénales, lesquelles ouvrent des procédures pénales contre ces personnes,

38 Cf. les constatations dans le Message du 19 février 2014 concernant la loi sur le renseignement (FF 2014 2029), p. 2040.

39 Ibid., p. 2044.

40 Ibid., p. 2067.

41 Cf. la plateforme TETRA (pour TErrorist TRAcking), laquelle est dirigée par Fedpol et qui rassemble le Service de renseignement de la Confédération (SRC), le Ministère public de la Confédération (MPC), le Corps des gardes-frontière (Cgfr) et autres autorités. Les objectifs de TETRA s'inscrivent dans la Stratégie de la Suisse pour la lutte contre le terrorisme, approuvée par le Conseil fédéral en 2015.

42 Cf. COSTER VAN VOORHOUT (n. 37) pour l'analyse du problème dans d'autre pays européens comme les Pays-Bas et l'Angleterre. 
par exemple pour transgression à la Loi fédérale interdisant les groupes « Al-Qaïda » et « Etat islamique » et les organisations apparentées du 12 décembre $2014^{43}$, la Loi fédérale sur les armes, les accessoires d'armes et les munitions du 20 juin $1997^{44}$, voire les art.135, 259 ou 260ter CP. Il s'ensuit l'ordonnance de mesures de contrainte comme la perquisition du domicile, le séquestre des objets et des documents, la surveillance téléphonique rétroactive ou en temps réel, la détention provisoire, l'audition de personnes ${ }^{45}$.

La transmission de telles informations par le SRC aux autres autorités est en effet prévue par la LRens, révisée et entrée en vigueur en septembre 2017. Même la transmission des données provenant de mesures de recherche soumises à autorisation est admise lorsque « ces données comportent des indices fondés relatifs à une infraction dont la poursuite peut donner lieu à une mesure de surveillance comparable en vertu du droit de procédure pénale » (art. 60 al. 3 LRens). On reconnait ainsi facilement la clause de la présomption de l'autorisation hypothétique - vague au maximum elle aussi - de l'art.141 al. 4 CPP. En principe, l'al. 4 de l'art. 60 LRens oblige le SRC à indiquer la provenance des données aux autorités de poursuite pénale. Mais, selon la jurisprudence il faut respecter le fait que les sources du SRC et d'autres services du renseignement étrangers soient protégées. Selon nos juges fédéraux, dévoiler ces sources afin de pouvoir vérifier, en tant que juge, les informations communiquées serait ainsi superflu car le SRC est soumis à un contrôle parlementaire et administratif, qui consiste notamment à vérifier la légalité des activités des services de renseignement. Un tribunal serait donc tout bonnement en droit de supposer que les informations contenues dans un tel rapport sont d'origine légale et il peut se dispenser de contrôler la licéité de la recolte de l'information ${ }^{46}$. En revanche, le Tribunal fédéral semble être plus sceptique envers des informations recueillies par une autorité pénale étrangère lors d'une enquête pénale étrangère, transmises par Interpol : ce n'est que

43 Loi fédérale interdisant les groupes «Al-Qaïda» et «Etat islamique» et les organisations apparentées du 12 décembre 2014 (RS 122).

44 Loi fédérale sur les armes, les accessoires d'armes et les munitions du 20 juin 1997 (RS 514.54).

45 Décision du tribunal pénal fédéral SK.2013.39 du 2 mai 2014 et rec tification du 22juillet 2014 ; Décision du tribunal fédéral 6B_57/2015 du 27 janvier 2016 ; Décision du tribunal fédéral 1B_487/2019 du 11 novembre 2019 - recours contre l'arrêt de la Cour de justice du canton de Genève, Chambre pénale de recours ACPR/654/2019 P/13548/2019 du 30 août 2019; Décision du tribunal pénal fédéral SK.2019.63 du 18 décembre 2019.

46 Décision du tribunal pénal fédéral SK.2013.39 du 2 mai 2014 et rectification du 22 juillet 2014, consid. 2.7 et décision du tribunal fédéral 6B_57/2015 du 27 janvier 2016 consid. 3.2.2, les deux arrêts sur la base du cadre législatif avant l'entrée en vigueur de la LRens ; cf. pour la base légale actuelle les art. 79 ss. LRens. dans un tel contexte qu'il a renvoyé l'affaire à la juridiction inférieure pour que le prévenu soit acquitté ${ }^{47}$.

Il s'agit dès lors d'une démarcation vague plus inquié- 42 tante encore que la première mentionnée, qui peut être observée à l'interface entre une procédure pénale et l'enquête menée par le SRC.

\section{c) Les éléments recueillis par les personnes privées à l'interne des entreprises}

Le troisième domaine délicat concerne les sources d'in- 43 formation privées : comment et dans quelles conditions l'intégration des informations recueillies par des personnes privées lors d'une enquête interne à une entreprise puisse s'effectuer.

Les sources d'information privées sont devenues d'un 44 intérêt énorme lorsqu'il s'agit notamment d'enquêtes dirigées à l'encontre des entreprises en matière de droit pénal économique ${ }^{48}$. En effet, le seul fait de parvenir à comprendre la structure d'une entreprise prévenue, en particulier s'il s'agit d'une multinationale, représente un grand défi pour le Ministère public. D’une part, recueillir et analyser ensuite tous les éléments potentiellement en rapport avec l'affaire, excède souvent les ressources humaines et financières d'un Ministère public. D'autre part, dans le cadre de procédures pénales simplifiées ou de procédures de l'ordonnance pénale, une entreprise prévenue est, cas échéant, disposée à coopérer, à soumettre aux autorités pénales volontairement des rapports ainsi que d'éventuels éléments supplémentaires issus de l'enquête interne, car un tel comportement est le moyen le plus prometteur pour parvenir à un accord de procédure ${ }^{49}$. Ainsi, la justice pénale s'est adaptée et le Tribunal fédéral montre une grande tolérance dans l'admission des rapports « internes »d'une entreprise faisant l'objet d'une enquête pénale - tolérance qui est justifiée pour des raisons d'efficacité et de proportionnalité ${ }^{50}$. Ces rapports sont « internes » dans le sens où ils sont le résultat des enquêtes généralement menées par le service

47 Décision du tribunal fédéral 6B_323/2019 du 5 février 2020 consid.1.4

48 MICHAEL MRÁZ, Kuckuckseier im Strafprozess « Interne Untersuchungen », FP numéro spécial 2020, p.170 ss ; DAVID MÜHLEMANN, Fairness und Verwertbarkeit unternehmensinterner Untersuchungen, PJA 2018, p. 468 ss. ; WOLFGANG WOHLERS / VERONICA LYNN, Das Anwaltsgeheimnis bei internen Untersuchungen, recht 2018, p. 17 ss (Commentaire de la décision du tribunal fédéral 1B_85/2016 du 20 septembre 2016).

49 OLIVIER THORMANN, Sicht der Strafverfolgung - Chancen \& Risiken, in: Romerio/Bazzani (édit.), Interne und regulatorische Untersuchungen II, Zurich 2016, p. 100 s. ; DAVID MÜHLEMANN, Unternehmensinterne Untersuchungen und strafprozessuale Verwertbarkeit von Mitarbeiterbefragungen, Zurich/St-Gall 2018, p.117.

50 Décision du tribunal pénal fédéral BB.2018.139 du 25 avril 2019 consid. 5.2 ; ATF 142 IV 207 consid. 7.2.2.1. Dans d'autres situations, un tel rapport peut aussi être saisi par le MP au cours d'une perquisition (cf. p.ex. ATF 142 IV 207). 
juridique ou de conformité (compliance) de l'entreprise concernée ou également par des cabinets d'audit ou des études d'avocats ${ }^{51}$.

En appréciant l'utilité de tels éléments recueillis par des employés ou mandataires externes de l'entreprise prévenue, il est proposé - dans l'intérêt de l'efficacité de la procédure - que ces éléments soient pris en considération par les autorités pénales et que celles-ci s'abstiennent même de procéder à des enquêtes supplémentaires de leur propre chef si les résultats de l'enquête interne semblent compatibles avec les conclusions de l'enquête pénale ${ }^{52}$. vée la qualité de preuve, il faut noter que la jurisprudence n'est en soi pas cohérente : en effet, le Tribunal fédéral, bien que refusant la qualité de preuve, est en même temps d'avis qu'un tel élément doit toutefois être évalué, quelles que soient son origine et sa désignation ${ }^{54}$. Ainsi, selon un avis plus nuancé, la qualification en tant que preuve devrait évidemment être admise, mais c'est l'exploitation ultérieure qui devrait être effectuée de manière particulièrement prudente ${ }^{55}$. On constate dès lors que la doctrine focalise son analyse des expertises privées sur la question de l'exploitation et non celle d'administration en tant que preuve.

51 DAMIAN K. GRAF, Interne Untersuchungen als Grundlage rascher Verfahren, in: Ackermann/Hilf (édit.), Kurzer Prozess, zu kurzer Prozess - im Wirtschaftsstrafverfahren, EIZ - Europa Institut Zürich Band/Nr. 191, Zürich 2019, p. 96 s. ; DAMIAN K. GRAF, Beschlagnahmefähigkeit von Befragungsprotokollen und Ermittlungserzeugnissen interner Untersuchungen, FP 6/2015, p. 346 s ; MÜHLEMANN (n.48), p. 477. Ainsi, l'établissement des faits est perçu par une partie de la doctrine d'être une activité forensique typique des avocats et ne pas une activité exclusivement attribuée aux autorités pénales selon les art. 139 al. 1 cum 311 al. 1 CPP, cf. GRAF, p. 100 et les renvois indiqués.

52 THORMANN (n.49), p.124.

53 Dans ce sens : Décision du tribunal fédéral 6B_48/2020 du 26 mai 2020 consid. 5.3.

54 Décision du tribunal fédéral 6B_272/2012 du 29 octobre 2012 consid. 2.3 ; Décision du tribunal fédéral 6B 48/2009 du 11 juin 2009 consid. 4.2 ; Décision du tribunal fédéral 6B_283/2007 du 5 octobre 2007 consid. 2

55 SCHMID/JOSITSCH-Praxiskommentar, art. $182 \mathrm{~N} 7$; Bâle-Campagne, Tribunal cantonal 4602018374 du 9 avril 2019 consid. 2.
Il se pose cependant la question de savoir si le rapport 49 interne d'une entreprise prévenue de corruption ou de blanchiment d'argent ou d'autres infractions peut vraiment être assimilé à une expertise privée. Bien qu'il soit probable que de tels rapports expliquent des méthodes d'affaires ${ }^{56}$ ou puissent guider l'interprétation de certaines transactions, on ne saurait attribuer d'emblée cette qualité d'expertise à un tel rapport.

Il y a plusieurs alternatives : une serait de qualifier le 50 rapport fourni avec le consentement de l'entreprise prévenue comme pièce à conviction au sens de l'art.192 CPP, ce qui impliquerait qu'il pourrait être ultérieurement exploité librement.

On pourrait aussi songer à l'application par analogie de 51 l'art. 160 CPP avec l'effet que le ministère public ou le tribunal devront s'assurer de la crédibilité et demander, cas échéant, des précisions.

Une troisième alternative serait, finalement, l'applica- 52 tion par analogie de l'art. $145 \mathrm{CPP}$ selon lequel l'autorité pénale peut, en lieu et place d'une audition ou en complément de celle-ci, inviter le comparant à lui présenter un rapport écrit sur ses constatations.

Évidemment, il n'est par ailleurs pas non plus exclu que 53 l'entreprise prévenue fasse tout simplement l'exercice spontané de son droit d'être entendu selon l'art.107 al. 1 let. d CPP.

En fait, même si le rapport d'une enquête interne décrit 54 un certain état de fait et expose des mesures d'instruction qui ont été mises en œuvre au sein de l'entreprise, il ne s'agit pas d'une enquête menée par les autorités pénales et les éléments ainsi recueillis ne sont pas, par conséquent, " propres à établir la vérité » sans autre formalité au sens de l'art.139 al. 1 CPP57. Même si l'enquête est déléguée à une étude d'avocats ou un cabinet de révision externe, le degré d'indépendance serait à vérifier par les autorités pénales et en cas de doute, il appartiendrait à l'autorité pénale de confirmer ou infirmer certains éléments du rapport par d'autres pièces à conviction ou par des auditions.

56 Par exemple un rapport pour la FIFA présentant le développement et le système complexe de billetterie, cf. Décision du tribunal pénal fédéral BB.2018.139 du 25 avril 2019 consid. 5.3 et 5.4.

57 Autre avis : DAVID RAEDLER, Les enquêtes internes dans un contexte suisse et américain, Instruction de l'entreprise ou Cheval de Troie de l'autorité ?, Lausanne 2018 (=CEDIDAC 101), p. 602. 


\section{Conclusion}

Notre analyse nous amène à la conclusion que le Code de procédure pénale contient un cadre légal peu clair en matière du droit de la preuve. Bien qu'on puisse comprendre que le législateur ait opté pour une approche pragmatique, guidé par l'envie de ne pas régler la matière trop en détails et d'éviter trop de formalisme, il a transgressé, à notre sens, les limites de l'acceptable sous l'angle des grands principes de base : l'équitabilité de la procédure pénale et la sécurité juridique.

Le problème fondamental réside dans le fait que l'on accorde d'un point de vue dogmatique, législatif et jurisprudentiel trop peu d'attention à l'action normative de transformation ou de qualification des éléments recueillis en preuve. Ainsi, la distinction entre l'administration et l'exploitation des preuves passe du flou à l'obscurité totale comme nous avons tenté de le démontrer, d'un point de vue dogmatique d'abord, puis en pratique par rapport à trois domaines spécifiques.

57 Nous avons tenté d'introduire un concept plus clair en mettant en avant la distinction appliquée par le Code de procédure pénale. Après la récolte des éléments provenant d'une pluralité de sources, il faut qu'ils soient éva- lués selon les critères prescrits aux art.139, l'art. 76 al. 1 en lien avec l'art. 100 al. 1 CPP. S'ils satisfont ces critères, la qualité de preuve leur sera accordée. Dans l'hypothèse inverse, il ne s'agit pas d'une preuve tout court - par exemple si la documentation de son existence et sa production font défaut. Le juge ne peut s'appuyer sur un tel élément pour établir la vérité matérielle comme ce serait le cas pour une preuve.

Selon notre perception, la conséquence liée au refus 58 d'accorder le statut de preuve comparée avec la conséquence liée à la décision d'inexploitabilité pourrait se manifester procéduralement : alors que la question d'inexploitablité d'une preuve est reservée au juge du fond ${ }^{58}$, la question d'attribution de qualité de preuve pourra être jugée définitevement par l'instance de recours.

Or, l'attribution de qualité de preuve n'implique pas, à ce 59 stade, qu'elle soit exploitable. Pour arriver à cette conclusion, il faut appliquer le contrôle prévu par le législateur en vertu de l'art.141 CPP. Ce n'est qu'après constatation de son exploitabilité que la preuve est apte à établir la vérité matérielle.

58 JEANNERET/KUHN (n. 6), N9015 s. 